\title{
LRS Bianchi Type -V Cosmology with Heat Flow in Scalar -Tensor Theory
}

\author{
C.P. Singh* \\ Department of Applied Mathematics, \\ Delhi College of Engineering, \\ Bawana Road, Delhi-110 042, India.
}

(Received on 17 July, 2008)

\begin{abstract}
In this paper we present a spatially homogeneous locally rotationally symmetric (LRS) Bianchi type -V perfect fluid model with heat conduction in scalar tensor theory proposed by Saez and Ballester. The field equations are solved with and without heat conduction by using a law of variation for the mean Hubble parameter, which is related to the average scale factor of metric and yields a constant value for the deceleration parameter. The law of variation for the mean Hubble parameter generates two types of cosmologies one is of power -law form and second the exponential form. Using these two forms singular and non -singular solutions are obtained with and without heat conduction. We observe that a constant value of the deceleration parameter is reasonable a description of the different phases of the universe. We arrive to the conclusion that the universe decelerates for positive value of deceleration parameter where as it accelerates for negative one. The physical constraints on the solutions of the field equations, and, in particular, the thermodynamical laws and energy conditions that govern such solutions are discussed in some detail.The behavior of the observationally important parameters like expansion scalar, anisotropy parameter and shear scalar is considered in detail.
\end{abstract}

Keywords: Cosmology; Bianchi type models; Hubble parameter; Deceleration parameter; Inflationary model; Heat flow

\section{INTRODUCTION}

The scalar tensor theories are the generalizations of Einstein's theory of gravitation in which the metric is generated by a scalar gravitational field together with non -gravitational field (matter). The scalar gravitational field itself is generated by the non -gravitational fields via a wave equation in curved space time. The strength of the coupling between gravity and scalar field is determined by an arbitrary coupling function $\omega$. The theories of gravitation involving scalar fields have been extensively developed by some authors [1-3]. There are two different types of gravitational theories involving a classical scalar field function $\phi$. Brans -Dicke[1] introduced a scalar -tensor theory of gravitation involving a scalar function in addition to the familiar general relativistic metric tensor. In this theory the scalar field has a dimension of inverse of the gravitational constant $G$ and its role is confined to its effects on gravitational field equations. The theory of second type involves a dimensionless scalar field, e.g., Saez -Ballester theory [4].

Saez and Ballester [4] developed a scalar -tensor theory in which the metric is coupled with a dimensionless scalar field in a simple manner. This coupling gives a satisfactory description of weak fields. In spite of a dimensionless character of the scalar field, an anti -gravity regime appears. This theory suggests a possible way to solve the Friedmann -Robertson -Walker (FRW) cosmologies. FRW and spatially homogeneous anisotropic models have been widely studied in this theory of gravitation. Singh and Agrawal [5,6], Ram and Singh [7], Ram and Tiwari [8], Singh and Ram [9], Reddy and Rao [10], Reddy [11], Mohanty and Sahu[12], Reddy et al.[13], and Rao et al.[14, 15] are some of the authors who have investigated the cosmological models in this theory.

The Bianchi type -V locally rotationally symmetric cosmologies (LRS type -V) have commanded the attention of

*Electronic address: cpsphderediffmail.com many researchers during the past decade. The reason is that, while their dynamical behavior can be far more general than the simple Robertson -Walker and Bianchi type -I models, they are still less complicated than the Bianchi types VI -IX. The study of Bianchi type $-\mathrm{V}$ cosmological models creates more interest as these models contain isotropic special cases and permit arbitrarily small anisotropic levels at certain stages. These properties make the models suitable as models of our universe.

In general relativity theory cosmological models are usually constructed under the assumption that the matter content of the universe is satisfactorily described by a perfect fluid. While this assumption may be a good approximation to the actual matter content of the universe at the present epoch, effects such as heat conduction and magnetic fields may not be negligible at earlier epochs of the evolution of the universe. As the matter is not expected to attain thermal equilibrium in the early stages of the evolution of the universe, it is evident that there would be heat flow during certain stages at least. The effect of heat flow in its early stages has been investigated by several authors like Roy and Prasad[16] studied LRS Bianchi -V models, which are of embedding class one filled with perfect fluid along with heat conduction and radiation. Vanleeumen et al.[17], Novello and Reboucas [18],Reboucas [19],Coley and Tupper [20, 21] Sviestins [22], Mukherjee [23], Banerjee and Sanyal [24], Deng [25] all have studied the cosmological models with heat conduction. Coley [26] and Coley and Hoogen [27] investigated LRS Bianchi -V imperfect fluid cosmology with and without heat conduction in which the equation of state are completely 'dimensionless'. Chan [28] developed an analytical solution for a collapsing radiating body consisting of an isotropic fluid with shear undergoing radial heat flow with outgoing radiation. Roy and Banerjee [29], and Singh [30] has studied the cosmological models with heat conduction and discussed its effect on the early stages of evolution of the universe.

It is the purpose of the present paper is to study LRS Bianchi type $-\mathrm{V}$ cosmological models in which the source of 
the gravitational field is a perfect fluid and heat conduction in scalar tensor theory proposed by Saez and Ballester. More precisely, in this paper we will investigate a law of variation for the mean Hubble parameter that yields a constant value for the deceleration parameter in a perfect fluid LRS Bianchi type $-\mathrm{V}$ cosmological models with and without heat flow. The law of variation for the mean Hubble parameter yields a new approach for solving field equations of cosmological models, i.e., an approach which is quite general and suitable for description of the present day universe. The feature of this law of variation of greatest interest is that it generates two forms of average scale factor (power -law and exponential forms) in terms of cosmic time $t$, which are used to solve Einstein's field equations explicitly. This will enable us to analyze the physical and kinematical behaviors of cosmological models in a straight forward manner.

Exact solutions that correspond to singular and non -singular models are found with and without heat flow. We also relate the heat conduction to the temperature and discuss thermodynamic questions raised by the explicit solutions. The energy conditions have been checked for the solutions. The behavior of observationally important parameters like expansion, shear and anisotropy parameter is considered in detail.

\section{MODEL AND FIELD EQUATIONS}

We consider a locally rotationally symmetric (LRS) Bianchi type $-\mathrm{V}$ space time with metric

$$
d s^{2}=-d t^{2}+A^{2}(t) d x^{2}+e^{2 x} B^{2}(t)\left(d y^{2}+d z^{2}\right),
$$

where $A(t)$ and $B(t)$ are the cosmic scale functions.

The energy -momentum tensor considered in this work, is due to a perfect fluid with heat conduction, viz.,

$$
T_{\mu v}=(\rho+p) u_{\mu} u_{v}+p g_{\mu v}+h_{\mu} u_{v}+h_{v} u_{\mu} .
$$

where $p$ is the thermodynamic pressure, $\rho$ the energy density, $u_{\mu}$ the four-velocity of the fluid and $h_{\mu}$ is the heat flow vector satisfying

$$
h^{\mu} h_{\mu}>0, \quad h^{\mu} u_{\mu}=0 .
$$

Let us consider that in Bianchi type $-\mathrm{V}$ models under consideration the fluid four velocity is co-moving i.e., $u^{\mu}=\delta_{0}^{\mu}$, then the field equations and Eq. (3) imply that the heat flow is in $x$-direction only so that $h_{\mu}=\left(0, h_{1}(t), 0,0,\right)$.

The field equations in the scalar-tensor theory, proposed by Saez and Ballester [4], are given by

$$
G_{\mu v}-\omega \phi^{r}\left(\phi_{, \mu} \phi_{, v}-\frac{1}{2} g_{\mu v} \phi_{, k} \phi^{, k}\right)=T_{\mu v}
$$

where $G_{\mu v}=R_{\mu v}-\frac{1}{2} g_{\mu \nu} R$ is the Einstein tensor and $8 \pi G=c=1$. The scalar field $\phi$ satisfies the equation

$$
2 \phi^{r} \phi_{; \mu}^{, \mu}+r \phi^{r-1} \phi_{, k} \phi^{, k}=0 .
$$

Here $r$ is an arbitrary constant and $\omega$ is a dimensionless coupling constant. Comma and semi-colon denote ordinary and covariant derivative with respect to cosmic time $t$, respectively.

The equation of motion is given by

$$
T_{; v}^{\mu v}=0
$$

We recall, that for Bianchi $-\mathrm{V}$ models under consideration, the average scale factor $a$ is defined as

$$
a=\left(A B^{2}\right)^{1 / 3},
$$

where volume scale factor $V$ is given by

$$
V=a^{3} \text {. }
$$

We define the generalized mean Hubble parameter $H$ as

$$
H=\frac{1}{3}\left(H_{1}+H_{2}+H_{3}\right),
$$

where $H_{1}=\dot{A} / A, H_{2}=H_{3}=\dot{B} / B$ are the directional Hubble parameters in the directions of $x, y$ and $z$ axes, respectively. A dot denotes a derivative with respect to cosmic time $t$. The physical quantities of observational interest in cosmology are the expansion scalar $\theta$, the average anisotropy parameter $A p$ and the shear scalar $\sigma^{2}$. These are defined as :

$$
\begin{gathered}
\theta=u_{; \mu}^{\mu}=\left(\frac{\dot{A}}{A}+2 \frac{\dot{B}}{B}\right), \\
A p=\frac{1}{3} \sum_{i=1}^{3}\left(\frac{H_{i}-H}{H}\right)^{2},
\end{gathered}
$$

$$
\sigma^{2}=\frac{1}{2} \sigma_{\mu \nu} \sigma^{\mu \nu}=\frac{1}{3}\left(\frac{\dot{A}}{A}-\frac{\dot{B}}{B}\right)^{2} .
$$

From Eqs.(7) -(9), we obtain an important relation

$$
H=\frac{\dot{a}}{a}=\frac{1}{3}\left(\frac{\dot{A}}{A}+2 \frac{\dot{B}}{B}\right) .
$$

For Bianchi $-\mathrm{V}$ metric (1) and energy momentum tensor (2), the non -trivial components of field equations (4) for a co-moving fluid are

$$
\begin{gathered}
2 \frac{\ddot{B}}{B}+\frac{\dot{B}^{2}}{B^{2}}-\frac{1}{A^{2}}=-p+\frac{1}{2} \omega \phi^{r} \dot{\phi}^{2} \\
\frac{\ddot{A}}{A}+\frac{\ddot{B}}{B}+\frac{\dot{A}}{A} \frac{\dot{B}}{B}-\frac{1}{A^{2}}=-p+\frac{1}{2} \omega \phi^{r} \dot{\phi}^{2} \\
2 \frac{\dot{A} \frac{\dot{B}}{A} \frac{\dot{B}^{2}}{B}+\frac{3}{B^{2}}-\frac{1}{A^{2}}}{}=\rho-\frac{1}{2} \omega \phi^{r} \dot{\phi}^{2} .
\end{gathered}
$$


An equation that defines the only non -zero component of the heat conduction vector $h_{\mu}$ along the $x$-direction is given by

$$
2\left(\frac{\dot{B}}{B}-\frac{\dot{A}}{A}\right)=h_{1}
$$

Equation (5) gives

$$
\ddot{\phi}+\dot{\phi}\left(\frac{\dot{A}}{A}+2 \frac{\dot{B}}{B}\right)+\frac{r}{2} \frac{\dot{\phi}^{2}}{\phi}=0
$$

From the above equations we obtain the 'generalized Friedmann equation'

$$
\rho=3 H^{2}-\sigma^{2}-\frac{3}{A^{2}}+\frac{1}{2} \omega \phi^{r} \dot{\phi}^{2} .
$$

From Eqs.(14) and (15), we obtain

$$
p=H^{2}(2 q-1)-\sigma^{2}+\frac{1}{A^{2}}+\frac{1}{2} \omega \phi^{r} \dot{\phi}^{2}
$$

where $q$ is the deceleration parameter, which will be defined later. Finally, the law of energy conservation equation (6) gives

$$
\dot{\rho}+(\rho+p)\left(\frac{\dot{A}}{A}+2 \frac{\dot{B}}{B}\right)=-\frac{2}{A^{2}} h_{1},
$$

\section{SOLUTION OF FIELD EQUATIONS}

The matter density $\Omega$ and the deceleration parameter $q$ are the most important observational parameters in cosmology. The values of the deceleration parameter separate decelerating $(q>0)$ from accelerating $(q<0)$ periods in the evolution of the universe. Determination of the deceleration parameter from the count magnitude relation for galaxies is a difficult task due to the evolutionary effects. The present value $q_{0}$ of the deceleration parameter obtained from observations are $-1.27 \leq q_{0} \leq 2$ (Schuecker et al.[31]). Studies of galaxy counts from redshift surveys provide a value of $q_{0}=0.1$, with an upper limit of $q_{0}<0.75$ (Schuecker et al.[31]). Recent observations show that the deceleration parameter of the universe is in the range $-1 \leq q \leq 0$, and the present day universe is undergoing accelerated expansion ( Riess et al.[32]; Perlmutter et al.[33, 34]). It may be noted that though the current observations of SNe Ia and the CMBR favour accelerating models $(q<0)$, they do not altogether rule out the existence of a decelerating phase during the early history of our universe, which are also consistent with these observations [35].

Cosmological models with the constant deceleration parameter have been intensively investigated in the physical literature. It has been shown by Berman [36], and Berman and Gomide [37] that all the phases of the universe, radiation and pressure -free phase, may be considered as the particular cases of the constant deceleration parameter type. Incidentally, most of the well -known perfect fluid models in general relativity and Brans -Dicke theory are studied with constant deceleration parameter. The theory of constant deceleration parameter in FRW model have been further studied by several authors [38-40]. Rahaman et al. [41] and Reddy et al.[13] have studied LRS Bianchi type -I models with negative deceleration parameter. In a series of recent papers, Singh and Kumar [42-44] and Kumar and Singh [45, 46] have extended the work to anisotropic Bianchi type -I and II cosmological models and obtained the solutions of field equations in general relativity and some scalar tensor theories.

In this paper we extend the work to obtain exact solutions of the field equations (14) -(18), which include heat conduction by using a law of variation for the mean Hubble parameter that yields a constant value for the deceleration parameter. Since the line element (1) is completely characterized by the mean Hubble parameter $H$, let us consider that the generalized mean Hubble parameter $H$ is related to the average scale factor $a$. According to the law, the variation for the mean Hubble parameter is given by

$$
H=l a^{-n}=l\left(A B^{2}\right)^{-n / 3},
$$

where $l(>0)$ and $n(\geq 0)$ are constants, and

$$
n=q+1 \text {, }
$$

where $H$ is defined as in Eq.(13) and $q$ is the deceleration parameter, defined as

$$
q=-\frac{\ddot{a} a}{\dot{a}^{2}} .
$$

Using Eqs.(22) and (23), the solution of Eq.(24) gives the law of variation of the average scale factor of the forms

$$
a=(n l t)^{1 / n}
$$

for $n \neq 0$ and

$$
a=a_{0} \exp \left(H_{0} t\right)
$$

for $n=0$, where $a_{0}$ is a constant of integration and $l=H_{0}$. Here, in Eq. (25) we have assumed that for $t=0$ the value $a=0$ so that the constant of integration turns out to be zero. Thus we have derived two types of models, depending upon whether $n \neq 0$ or $n=0$. It is however possible to have $l=0$ in Eq. (22) for which we would have a static universe. Hence we disregard a static universe. But $l>0$ is consistent with observation for which the universe must be expanding. The sign of $q$ indicates whether the model inflates or not. A positive sign of $q$, i.e., $(n>1)$ corresponds to "standard" decelerating model whereas the negative sign $-1 \leq q<0$ for $0 \leq n<1$ indicates inflation. The present day universe has been thought of as Einstein -de Sitter, with a constant deceleration parameter $q=1 / 2$. Recent observations of Supernovae data [32- 34] confirm that the universe is accelerating,therefore we may ask whether the value of the deceleration parameter could be different from the de Sitter universe, 
say as defined in equation (23). In the case of an accelerating universe, the second case for $n=0$ becomes very relevant. More over, it should be noted that this formula independent of the particular gravitational theory being considered.

\section{CASE (I): SOLUTION WITHOUT HEAT CONDUCTION $\left(h_{1}=0\right)$}

In this case, Eq. (17) gives

$$
A=k B
$$

where $k$ is a constant of integration. In this scenario the shear scalar and anisotropic parameter are zero and the field equations (14)- (16), or equivalent Eqs.(19) and (20) reduce to two independent equations for $\rho$ and $p$.

$$
\begin{gathered}
\rho=3 H^{2}-\frac{3}{k^{2} B^{2}}+\frac{1}{2} \omega \phi^{r} \dot{\phi}^{2} \\
p=H^{2}(2 q-1)+\frac{1}{k^{2} B^{2}}+\frac{1}{2} \omega \phi^{r} \dot{\phi}^{2}
\end{gathered}
$$

Using (27), the first integral of Eq. (18) gives

$$
\phi^{r / 2} \dot{\phi}=\frac{\phi_{0}}{B^{3}}
$$

where $\phi_{0}$ is a constant of integration. The conservation Eq. (21) reduces to

$$
\dot{\rho}+3(\rho+p)\left(\frac{\dot{B}}{B}\right)=0,
$$

The field equations (28) -(30) are a system of three equations with four unknown variables, namely, $B, \rho, p$ and $\phi$. For complete determinacy of the system one extra condition is needed. Now, we shall discuss the models for two cases depending upon the values of $n$ as defined in Eqs.(25) and (26).

\section{POWER -LAW SOLUTION WHEN $n \neq 0$}

In this case, from Eqs.(7), (25) and (27), we get the solution of scale factors as

$$
\begin{gathered}
B(t)=k^{1 / 3}(n l t)^{1 / n} \\
A(t)=k^{2 / 3}(n l t)^{1 / n} .
\end{gathered}
$$

Using Eq.(32) in Eq.(30) and integrating, we get

$$
\phi^{\frac{r+2}{2}}=\phi_{1}+\frac{\phi_{0} k}{2 l} \frac{(r+2)}{(n-3)}(n l t)^{\frac{n-3}{3}}
$$

where $\phi_{1}$ is a constant of integration and $n \neq 3$.

The expansion scalar is given by

$$
\theta=3(n t)^{-1}
$$

Here, we discuss the solutions for non -flat and flat models. Non -flat models :

In this case the Ricci scalar is given by

$$
R=6\left[(2-n)(n t)^{-2}-k^{-4 / 3}(n l t)^{-2 / n}\right] .
$$

Using Eqs.(32)-(35) in Eqs.(28) and (29), the energy density and pressure are, respectively, given by

$$
\begin{gathered}
\rho=3(n t)^{-2}-3 k^{-4 / 3}(n l t)^{-2 / n}+\frac{1}{2} \omega \phi_{0}^{2} k^{2}(n l t)^{-6 / n} \\
p=(2 n-3)(n t)^{-2}+k^{-4 / 3}(n l t)^{-2 / n}+\frac{1}{2} \omega \phi_{0}^{2} k^{2}(n l t)^{-6 / n} .
\end{gathered}
$$

The above set of solutions identically satisfy the conservation equation (31). In this scenario the average anisotropy parameter and shear scalar are zero, and the model involves at most energy density and pressure. A simple analysis in this model shows that $A(t)$ and $B(t)$ are positive and increasing function of cosmic time $t$, and the model is expanding ( i.e $\theta>0$ ). The expansion parameter $H_{\mu}$ in the directions of coordinates axes $(x, y, z)$ are same, i.e., $H_{1}=H_{2}=H_{3}=(n t)^{-1}$. The model represents non -shearing, non -rotating isotropic and conformally flat universe. The model starts expanding with Big -Bang at $t=0$, which is a point singularity of the model. The model exhibits power -law expansion after Big -Bang. The Ricci Scalar, energy density and pressure tend to infinity at $t=0$ and $R \rightarrow 0, \rho \rightarrow 0$, and $p \rightarrow 0$ as $t \rightarrow \infty$. Therefore, the model would give essentially empty universe at large time. The spatial volume tends to infinity as $t \rightarrow \infty$. The rate of expansion in the model stops when $t \rightarrow \infty$. We observe that $\lim _{t \rightarrow \infty} \rho / \theta^{2}=$ constant for $0<n<1$, which shows that the universe remains homogeneous with time. Since $d \theta / d t<0$, we conclude that the model starts expanding from its singular state and the rate of expansion decreases to zero as $t \rightarrow \infty$.

Flat Models :

In this case, Ricci scalar, $R=0$ and hence we get

$$
(2-n)(n t)^{-2}=k^{-4 / 3}(n l t)^{-2 / n} .
$$

The energy density and pressure have the following expression:

$$
\rho=3(n-1)(n t)^{-2}+\frac{1}{2} \omega \phi_{0}^{2} k^{2}(n l t)^{-6 / n}
$$

$$
p=(n-1)(n t)^{-2}+\frac{1}{2} \omega \phi_{0} k(n l t)^{-6 / n} .
$$

We find that the model starts expanding with the Big -Bang at $t=0$ as $\rho$ and $p$ both tend to infinity at this point. As $t \rightarrow \infty$, both $\rho$ and $p$ tend to zero. When energy density is large $(\rho \rightarrow \infty)$, the model corresponds to a radiation era and when density is small, $(\rho \rightarrow 0)$ the model corresponds to a vacuum phase. Therefore, in a generic situation, 
model can be interpolating between different phases of the universe: from a radiation universe with equation of state $p=\rho / 3$ to the vacuum phase. For $n=1$, we find the stiff matter $\operatorname{phase}(p=\rho)$. For the reality of $\rho \geq 0$ and $p \geq 0$, we must have $n \geq 1$. The model represents an expanding non-shearing and isotropic universe.

\section{EXPONENTIAL SOLUTION WHEN $n=0$}

In this case, from Eqs.(7),(26) and (27), we get the solution of scale factors as

$$
\begin{gathered}
A(t)=k^{2 / 3} a_{0} \exp \left(H_{0} t\right) \\
B(t)=k^{-1 / 3} a_{0} \exp \left(H_{0} t\right) .
\end{gathered}
$$

The scalar field is given by

$$
\phi^{\frac{r+2}{2}}=\phi_{2}+\frac{\phi_{0} k}{6 l} \frac{(r+2)}{a_{0}^{3}} \exp \left(-3 H_{0} t\right)
$$

The expansion scalar is given by

$$
\theta=3 H_{0}
$$

Here, we again discuss the solutions for non -flat and flat models.

\section{Non -flat models :}

In this case the Ricci scalar is given by

$$
R=6\left[2 H_{0}^{2}-k^{-4 / 3} a_{0}^{-2} \exp \left(-2 H_{0} t\right)\right]
$$

When $t=0, R=6\left(2 H_{0}^{2}-k^{-4 / 3} a_{0}^{-2}\right)$ and when $t \rightarrow \infty$, $R=12 H_{0}^{2}$.

The energy density and pressure are, respectively, given by

$$
\rho=3 H_{0}^{2}-3 k^{-4 / 3} a_{0}^{-2} \exp \left(-2 H_{0} t\right)+\frac{\omega \phi_{0}^{2} k^{2}}{2 a_{0}^{6}} \exp \left(-6 H_{0} t\right)
$$

$$
p=-3 H_{0}^{2}+k^{-4 / 3} a_{0}^{-2} \exp \left(-2 H_{0} t\right)+\frac{\omega \phi_{0}^{2} k^{2}}{2 a_{0}^{6}} \exp \left(-6 H_{0} t\right)
$$

The above solutions identically satisfy the conservation equation (31).

The pressure and energy density describe by a vacuum energy density $p=-\rho$ at later stage of evolution. The directional Hubble parameters are all constant and have equal value i.e. $H_{1}=H_{2}=H_{3}=H_{0}$ through out the evolution. The average anisotropy and shear scalar are zero. The ratio $\lim _{t \rightarrow \infty} \rho / \theta^{2}$ tends to constant, which shows that the universe remains homogeneous with cosmic time. The scale factors, energy density and pressure all are constant at $t=0$ and the rate of expansion is also constant through out the evolution. The model starts with a constant volume and expands exponentially with time. As time passes, volume and scale factors expand exponentially and the universe becomes infinitely large as $t \rightarrow \infty$. The negative pressure predicts an accelerating phase for which the deceleration parameter is negative.

\section{Flat models:}

For flat model $R=0$ and hence in this case, we have

$$
2 H_{0}^{2}=k^{-4 / 3} a_{0}^{-2} \exp \left(-2 H_{0} t\right)
$$

The energy density and pressure now take the form

$$
\begin{aligned}
& \rho=H_{0}^{2}\left(\omega \phi_{0} k^{10 / 3} a_{0}^{-4}-3\right) \\
& p=H_{0}^{2}\left(\omega \phi_{0} k^{10 / 3} a_{0}^{-4}-1\right)
\end{aligned}
$$

For energy density to be positive we must have $\omega \phi_{0} k^{10 / 3} a_{0}^{-4} \geq 3$.

\section{CASE II: SOLUTION WITH HEAT CONDUCTION $\left(h_{1} \neq 0\right)$}

In this case,the field equations (14) -(18) are a system of five equations with six unknown variables, namely, $A, B, \rho, p$, $h_{1}$ and $\phi$. In what follows, we try to solve the field equations (14) -(18) with heat conduction.

Subtracting (15) from (14), we get

$$
\frac{\ddot{B}}{B}-\frac{\ddot{A}}{A}+\frac{\dot{B}^{2}}{B^{2}}-\frac{\dot{A}}{A} \frac{\dot{B}}{B}=0,
$$

which on integration gives

$$
\frac{\dot{B}}{B}-\frac{\dot{A}}{A}=\frac{k_{1}}{A B^{2}}
$$

where $k_{1}$ is a constant of integration. Using Eq.(7) in Eq. (53) and integrating again, we get

$$
\frac{B}{A}=d_{1} \exp \left(\int \frac{k_{1}}{a^{3}} d t\right) .
$$

The quadrature form of metric functions $A$ and $B$ in terms of the average scale factor $a$ are given by

$$
\begin{gathered}
A(t)=\left(d_{1}\right)^{-2 / 3} a \exp \left(-\frac{2 k_{1}}{3} \int a^{-3} d t\right), \\
B(t)=\left(d_{1}\right)^{1 / 3} a \exp \left(\frac{k_{1}}{3} \int a^{-3} d t\right) .
\end{gathered}
$$

The first integral of Eq. (18) gives

$$
\phi^{r / 2} \dot{\phi}=\frac{\phi_{3}}{A B^{2}}
$$


where $\phi_{3}$ is a constant of integration. Now, we shall seek exact solutions (cosmological models) of Eqs. (55) and (56) and corresponding solutions of energy density and pressure from Eqs. (19) and (20) in the following two subsections, depending on the values of $n$ as defined in Eqs.(25) and (26).

\section{POWER -LAW SOLUTION}

In this case, using Eq.(25) in Eqs. (55) and (56) the solution of metric functions are given by

$$
\begin{aligned}
& A(t)=\left(d_{1}\right)^{-2 / 3}(n l t)^{1 / n} \exp \left[-\frac{2 k_{1}}{3 l(n-3)}(n l t)^{(n-3) / n}\right], \\
& B(t)=\left(d_{1}\right)^{1 / 3}(n l t)^{1 / n} \exp \left[\frac{k_{1}}{3 l(n-3)}(n l t)^{(n-3) / n}\right],
\end{aligned}
$$

where $n \neq 3$. From Eq. (17), the solution of heat conduction is given by

$$
h_{1}=2 k_{1}(n l t)^{-3 / n} .
$$

From (57), we get the solution of scalar field as

$$
\phi^{\frac{r+2}{2}}=\phi_{4}+\frac{\phi_{3}}{2 l} \frac{(r+2)}{(n-3)}(n l t)^{\frac{n-3}{3}}
$$

The directional Hubble parameters along $x, y$ and $z$ directions are given by

$$
\begin{gathered}
H_{1}=(n t)^{-1}-\frac{2 k_{1}}{3}(n l t)^{-3 / n}, \\
H_{2}=H_{3}=(n t)^{-1}+\frac{k_{1}}{3}(n l t)^{-3 / n},
\end{gathered}
$$

whereas the generalized mean Hubble parameter is given by

$$
H=(n t)^{-1} .
$$

The other important physical and geometrical parameters have the following expressions:

$$
\begin{gathered}
\theta=3(n t)^{-1} \\
A_{p}=\frac{2 k_{1}^{2}}{l^{2}}(n l t)^{(2 n-6) / n} \\
\sigma^{2}=k_{1}^{2}(n l t)^{-6 / n} .
\end{gathered}
$$

Using the above solutions in Eqs.(19) and (20), the energy density and pressure have the following form:

$$
\begin{aligned}
\rho= & 3(n t)^{-2}-k_{1}^{2}(n l t)^{-6 / n}-3\left(d_{1}\right)^{4 / 3}(n l t)^{-2 / n} \\
& \times \exp \left[\frac{4 k_{1}}{3 l(n-3)}(n l t)^{(n-3) / n}\right]+\frac{1}{2} \omega \phi_{3}^{2}(n l t)^{-6 / n}(68)
\end{aligned}
$$

$$
\begin{aligned}
p= & (2 n-3)(n t)^{-2}-k_{1}^{2}(n l t)^{-6 / n}+\left(d_{1}\right)^{4 / 3}(n l t)^{-2 / n} \\
& \times \exp \left[\frac{4 k_{1}}{3 l(n-3)}(n l t)^{(n-3) / n}\right]+\frac{1}{2} \omega \phi_{3}^{2}(n l t)^{-6 / \eta}(69)
\end{aligned}
$$

We find that the above solutions satisfy the conservation equation (21) identically. Thus, we have obtained an exact solution of the Bianchi type -V models explicitly.

From the above set of solutions we observe that the spatial volume is zero at $t=0$. The metric functions $A(t)$ and $B(t)$ are also zero at this initial value. The energy density and pressure become infinite at $t=0$. The physical parameters $\theta, A p$ and $\sigma^{2}$ are all infinite at this initial point. The model has a point singularity at $t=t_{1}$. The heat conduction is a decreasing function of time and is infinite at the initial epoch. As $t \rightarrow \infty$, both the scale factors $A(t)$ and $B(t)$ tend to indeterminate forms whereas $p$ and $\rho$ tend to zero. The other physical parameters such as $\theta, A p$ for $n<3$ and $\sigma^{2}$ tend to zero as $t \rightarrow \infty$, which indicates that the universe is expanding with cosmic time but the rate of expansion is decreasing and the model becomes isotropic for large times. Also, we find that $\lim _{t \rightarrow \infty} \sigma / \theta^{2}=0$ for $n<3$ and $\lim _{t \rightarrow \infty} \rho / \theta^{2}=$ const., which indicate that the models approach to isotropy for large $t$. The flow of heat along the $x$ - direction was maximum early on, and it diminishes as $t \rightarrow \infty$. We also observe that $\sigma^{2}=h_{1}^{2} / 4$, where we have used Eq. (59) to relate shear scalar and heat conduction, and as such we obtain $\sigma \propto h_{1}$.

\section{EXPONENTIAL SOLUTION}

In this case, using Eq.(26) in Eqs.(55) and (56), the solution of scale factors are given by

$$
\begin{gathered}
A(t)=\left(d_{1}\right)^{-2 / 3} a_{0} \exp \left[H_{0} t+\frac{2 k_{1}}{3 H_{0} a_{0}^{3}} \exp \left(-3 H_{0} t\right)\right], \\
B(t)=\left(d_{1}\right)^{1 / 3} a_{0} \exp \left[H_{0} t-\frac{k_{1}}{3 H_{0} a_{0}^{3}} \exp \left(-3 H_{0} t\right)\right] .
\end{gathered}
$$

The directional Hubble parameters are given by

$$
H_{1}=H_{0}-\frac{2 k_{1}}{3 a_{0}^{3}} \exp \left(-3 H_{0} t\right)
$$

$$
H_{2}=H_{3}=H_{0}+\frac{k_{1}}{3 a_{0}^{3}} \exp \left(-3 H_{0} t\right),
$$

whereas the generalized mean Hubble parameter is given by

$$
H=H_{0}
$$

The other physical parameters have the following expressions:

$$
\theta=3 H_{0},
$$

$$
A p=\frac{5}{3} \frac{k_{1}^{2}}{H_{0}^{2} a_{0}^{6}} \exp \left(-6 H_{0} t\right),
$$




$$
\begin{gathered}
\sigma^{2}=\frac{5}{2} \frac{k_{1}^{2}}{a_{0}^{6}} \exp \left(-6 H_{0} t\right), \\
q=-1 .
\end{gathered}
$$

The solution of heat conduction is given by

$$
h_{1}=\frac{2 k_{1}}{a_{0}^{3}} \exp \left(-3 H_{0} t\right) .
$$

The scalar field has the expression

$$
\phi^{\frac{r+2}{2}}=\phi_{5}-\frac{\phi_{3}}{6 H_{0}} \frac{(r+2)}{c_{2}^{3}} \exp \left(-3 H_{0} t\right)
$$

The energy density and pressure are respectively given by

$$
\begin{aligned}
\rho= & 3 H_{0}^{2}-\frac{5}{2} \frac{k_{1}^{2}}{a_{0}^{6}} \exp \left(-6 H_{0} t\right) \\
& -3\left(d_{1}\right)^{4 / 3} a_{0}^{-2} \exp \left[-2\left\{H_{0} t+\frac{2 k_{1}}{3 H_{0} a_{0}^{3}} \exp \left(-3 H_{0} t\right)\right\}\right] \\
& +\frac{1}{2} \omega \phi_{3}^{2} a_{0}^{-6} \exp \left(-6 H_{0} t\right) \\
p= & -3 H_{0}^{2}-\frac{5}{2} \frac{k_{1}^{2}}{a_{0}^{6}} \exp \left(-6 H_{0} t\right) \\
& +\left(d_{1}\right)^{4 / 3} a_{0}^{-2} \exp \left[-2\left\{H_{0} t+\frac{2 k_{1}}{3 l a_{0}^{3}} \exp \left(-3 H_{0} t\right)\right\}\right] \\
& +\frac{1}{2} \omega \phi_{3}^{2} a_{0}^{-6} \exp \left(-6 H_{0} t\right) .
\end{aligned}
$$

The above solutions identically satisfy the conservation equation (21). We observe that all the geometrical and physical parameters such as $A(t), B(t), V(t), \rho, p, \theta, A p, \sigma^{2}$ and the heat flow are constant at $t=o$. Thus the universe starts evolving with constant physical and geometrical parameters. The rate of expansion is uniform through out the evolution. As $t \rightarrow \infty, A(t)$ and $B(t)$ tend to infinity whereas $\rho$ and $p$ are related by the equation of state $p=-\rho$, which shows that the fluid behaves as a vacuum with energy density causing the acceleration of the universe. The mean anisotropic parameter and shear scalar vanish as $t \rightarrow \infty$. The heat conduction is a decreasing function of time and constant at $t=0$. The heat flow also becomes negligible for large times $t$. Thus, the model starts with a constant volume and expands exponentially with time. As time passes, the volume and scale factors expand exponentially and the universe becomes infinitely large as $t \rightarrow \infty$. The negative pressure predicts an accelerating phase for which deceleration parameter is negative.

\section{THE PHASES OF THE UNIVERSE}

$\operatorname{Gr} \phi \mathrm{n}$ [47] has remarked that it is current belief that the universe had the following early phases:

$$
a_{1} \propto t^{1 / 2}, \quad p=\rho / 3
$$

$$
\begin{array}{cc}
a_{2} \propto \exp (H t), & p=-\rho \\
a_{3} \propto t^{1 / 2}, & p=\rho / 3
\end{array}
$$

On the other hand, according to Schwarzschild [48], the matter-dominated phase of the universe is given by

$$
a_{4} \propto t^{2 / 3}, \quad p=0
$$

The current accelerated phase of the universe can be represented by

$$
a_{5} \propto t^{4 / 3}
$$

or, alternatively, by equation (84).

We have already discussed in previous sections that the law of variation for the Hubble parameter (22) yields a constant value for the deceleration parameter $n=q+1$ and generates two forms of cosmologies (25) and (26). We find out that the above five phases are particular cases of the constant deceleration parameter type. It is evident from equations (25) and (26) that $n=o$ stands for inflation, while $n=2, n=3 / 2$ and $n=3 / 4$ stand, respectively, for the first, third, fourth and fifth phases. A relation between pressure and energy density for $n \neq 0$ can be written as

$$
p=\left(\frac{2 n-3}{3}\right) \rho,
$$

which is the perfect gas law equation of state.

On the other hand, for $n=0$, we have

$$
p=-\rho
$$

Thus, we observed that for $n \neq 0$ phases, we have a perfect gas equation of state, while for the $n=0$ (inflation) case, we have negative pressure. The equation of state described by equation (88)can be taken to get the solutions for different phases of universe.

\section{THERMODYNAMICAL RELATIONS}

Now, we discuss the thermodynamical relations depending on the following thermodynamical laws $[49,50]$ with the solution of heat conduction obtained in Eqs. (60) and (79):

\section{Baryon conservation law:}

In standard cosmology, conservation of total particle number gives

$$
N_{; \mu}^{\mu}=0
$$

where $N^{\mu}=\chi u^{\mu}$ is the particle flux and $\chi$ is the particle density, which is given by

$$
\frac{d \chi}{d t}=-\chi \theta
$$


Using the power-law solutions $(n \neq 0)$ obtained in case II of section III in Eq. (91) and integrating, we get

$$
\chi=b_{1}(n l t)^{-3 / n},
$$

and using the exponential solutions $(n=0)$ in Eq.(91) and integrating, we get

$$
\chi=\frac{b_{2}}{c^{3}} \exp (-3 l t)
$$

where $b_{1}$ and $b_{2}$ are constants of integration. Using Eqs.(60) and (79), Eqs. (92) and (93) yield

$$
\chi \propto h_{1}
$$

In the case of $n \neq 0$, the particle density is large at $t=0$ so the heat conduction has a greater influence during early stages of evolution. In the case of $n=0$, the particle density is constant at $t=0$, which implies that heat conduction is constant. Both the Eqs. (92) and (93) are decreasing function of time.

\section{Temperature gradient law:}

The general relativistic expression for heat conduction [49] is given by

$$
h^{\mu}=-\kappa\left(g^{\mu v}+u^{\mu} u^{v}\right)\left(T_{, v}+T u_{v ; \alpha} u^{\alpha}\right),
$$

where $\kappa \geq 0$ is the heat conduction coefficient, i.e., thermal conductivity, $T$ is the temperature, and $u_{v ; \alpha} u^{\alpha}$ is the acceleration. Since in our case only the $x$-component of heat flux is retained, from above equation we obtain

$$
h_{1}=\kappa T_{; 1} .
$$

From the above equation and Eqs. (60) and (79), we can have the $x$-component of temperature gradient as: In case of $n \neq 0$, we have

$$
T_{; 1}=\frac{2 k_{1}(n l t)^{-3 / n}}{\kappa},
$$

and in case of $n=0$, we get

$$
T_{; 1}=\frac{2 k_{1} \exp (-3 l t)}{c^{3} \kappa},
$$

The homogeneity consideration restricts the thermal conductivity as a function of time alone so that $\kappa=\kappa(t)$. Integrating Eqs. (97) and (98), we get, respectively, the temperature distribution for $n \neq 0$ and $n=0$ in the forms:

$$
\begin{gathered}
T=\frac{k_{1}(n l t)^{-3 / n}}{\kappa(t)} x+\eta_{1}(t), \\
T=\frac{k_{1}}{c^{3}} \frac{\exp (-3 l t)}{\kappa(t)} x+\eta_{2}(t),
\end{gathered}
$$

where $\eta_{1}(t)$ and $\eta_{2}(t)$ appear as integration constant, which may either be an arbitrary functions of time or constants. We observe that $T$ diverges at the initial epoch as long as the coefficient of thermal conductivity remains finite. This is consistent with the situation in big bang cosmology. At the final stage of expansion $t \rightarrow \infty$, we have $T \rightarrow \eta_{1}(t)$ in case of $n \neq 0$ and $T \rightarrow \eta_{2}(t)$ in case of $n=0$, which implies that the universe will be in thermal equilibrium at the final stage of evolution.

\section{Entropy Production:}

The second law of thermodynamics suggests

$$
S_{; \mu}^{\mu} \geq 0
$$

where $S^{\mu}$ is the entropy flux defined by

$$
S^{\mu}=S u^{\mu}+T^{-1} h^{\mu}
$$

and $S$ is the entropy density, which is described by $S=s \chi$ and $s$ is the entropy per particle.

The entropy flux is given by

$$
S^{\mu}=\left(S, \frac{1}{T A^{2}} h_{1}, 0,0\right) .
$$

By a straightforward calculation, it is verified that $S_{; \mu}^{\mu} \geq 0$.

\section{CONCLUSION}

We have discussed a law of variation for the mean Hubble parameter that yields a constant value for the deceleration parameter and generates two types of cosmologies for the average scale factor. We have then considered these two forms of cosmologies to solve the field equations of LRS Bianchi type $-\mathrm{V}$ perfect fluid models with and without heat flow in Saez -Ballester's scalar tensor theory. We have obtained exact solutions in both with and without heat flow models. The solutions have been further classified into flat and non -flat models in the absence of heat conduction. In the cosmology with the power -law solution, we have obtained exact solutions which start evolving from its singular state and expanding model and the model always decelerates. At the initial epoch, the proper volume vanishes whereas all other parameters, including heat conduction and temperature diverse. The solution obtained from exponential form with a negative deceleration parameter corresponds to a non -singular state and expands exponentially with a constant rate of expansion scalar. All the physical parameters are constant. The heat conduction and temperature are decreasing functions of time and are maximum at early stages of evolution of the universe. The rate of expansion slows down and vanishes as $t \rightarrow \infty$. At the final stage of evolution, the proper volume takes infinitely large values while other parameters such as $\rho, p, \sigma^{2}$ etc, become insignificant. So, effectively, isotropy is not an initial condition in our model. We have started from an anisotropic universe and have shown how isotropization takes place with the evolution of the universe. We have also shown that at the final stage 
of the evolution, temperature $T=\eta(t)$ becomes spatially homogeneous, which implies that the universe attains thermal equilibrium everywhere. In the case of exponential variation of the average scale factor, we have presented an accelerating non-singular model of the universe. The model represents uniform expansion and the volume grows exponentially with cosmic time. The solutions help to resolve several cosmological problems associated with the standard model ( flatness, horizon, monopole etc). We have also discussed the physical and kinematical behavior of the models in two types of cosmologies. For a realistic distribution we require that the density be positive, the pressure be non -negative and that the velocity of sound does not exceed the velocity of light in the medium. A set of energy conditions given by Hawking and Ellis [51] are: (i) $\rho>0$, (ii) $p \geq 0$, (iii) $\rho+p \geq 0$ and (iv) $\rho+3 p \geq 0$. These energy conditions are identically satisfied provided $n>3 / 2$ in case of $n \neq 0$. The Hawking - Penrose energy condition [52] is not violated during the evolution of the model from zero proper volume to infinitely large proper volume, since $\rho$ is a decreasing function of time from infinitely large to vanishingly small values as the model expands.

The models presented in this paper could give an ap- propriate description of the evolution of universe. It is noted that the universe as described by the equation of state $p=-\rho$ displays de -Sitter behaviour in the case of $q=-1$. In general relativity, the vacuum energy density can be included in the cosmological constant term. This class of solutions is consistent with early inflation. A positive cosmological constant resists the attractive gravity of matter due to its negative pressure. For most models, the positive cosmological constant eventually dominates over the attraction of matter and drives the universe to expand exponentially. The heat conduction was large at early stage of evolution of universe and vanishes at later stage of its evolution. The models represent shearing, non -rotating and expanding in the presence of heat flow, which approach to isotropic for large values of cosmic time. But the models show no shear and anisotropy without heat conduction.

We have observed that this approach can lead to a better understanding of early universe, where the heat flow type physical contains play probably an important role in the evolution of universe. The constantly decelerating perfect fluid model with heat flow in an anisotropic geometry leads to a self consistent solution, which may describe a well -determined period of the evolution of our universe.
[1] C. Brans, and R.H. Dicke, Phys. Rev. 124, 925 (1961).

[2] P.G. Bergmann, Int.J.Theo.Phys. 1, 25 (1968).

[3] K. Nordvedt, Astrophys. J. 161, 1059 (1970).

[4] D. Saez and V.J. Ballester, Phys. Lett. A. 113, 467 (1985).

[5] T. Singh and A. K. Agarwal, Astrophys. Space. Sci.182, 289 (1991).

[6] T. Singh and A.K. Agarwal, Astrophys. Space. Sci. 191, 61 (1992).

[7] S. Ram and J.K. Singh, Astrophys. Space. Sci. 234, 325 (1995).

[8] S. Ram and S.K. Tiwari, Astrophys. Space. Sci. 259, 91 (1998).

[9] C.P. Singh and S. Ram, Astrophys. Space. Sci. 284, 1199 (2003).

[10] D.R.K. Reddy and Venkateswara Rao, Astrophys. Space. Sci. 277, 461 (2001).

[11] D.R.K. Reddy, Astrophys. Space. Sci. 286, 359 (2003).

[12] G. Mohanty and S.K. Sahu, Astrophys. Space. Sci. 288, 611 (2003).

[13] D.R.K. Reddy, R.M.V. Subba and R.G. Koteswara, Astrophys. Space Sci., 306, 171 (2006).

[14] V.U.M. Rao, T. Vinutha and M. Vijaya Shanthi, Astrophys. Space Sci. 312,189 (2007).

[15] V.U.M. Rao, M. Vijaya Shanthi and T. Vinutha, Astrophys. Space Sci.

314, 73 (2008).

[16] S. Roy and A. Prasad, Gen. Relativ. Gravit. 26, 939, (1994).

[17] W.A. Vanleeuwen, A.J. Kox and S.R. de Groot, Physica A 79, 233 (1975).

[18] M. Novello and M.J. Reboucas, Astrophys. J. 225, 719 (1978).

[19] M.J. Reboucas, IL Nuovo Cim. B 67, 120 (1982).
[20] A.A. Coley and B.O.J. Tupper, Phys. Lett. A 95, 357 (1983).

[21] A.A. Coley and B.O.J. Tupper, Astrophys. J. 280, 26 (1984).

[22] E. Sviestins, Gen. Relativ. Gravit. 17, 521 (1985).

[23] G. Mukherjee, J. Astrophys. Astron. 7, 259 (1986)

[24] A. Banerjee and A.K. Sanyal, Gen. Relativ. Gravit. 20, 103 (1988).

[25] Y. Deng, Gen. Relativ. Gravit. 21, 503 (1989).

[26] A.A. Coley, Gen. Relativ. Gravit. 22, 3 (1990); J. Math. Phys. 31 , 1698 (1990).

[27] A.A. Coley and R.J. Hoogen, J. Math. Phys. 35, 4117 (1994).

[28] R. Chan, Astrophys. Space Sci. 275, 299 (1998).

[29] S.R. Roy and S.K. Banerjee, Gen. Relativ. Gravit. 28, 27 (1996).

[30] J.K. Singh, Astrophys. Space Sci. 310, 241 (2007).

[31] R. Schuecker et al., Astrophys. J. 496, 635 (1998).

[32] A.G. Riess et al., Astrophys. J. 116, 1009 (1998).

[33] S. Perlmutter et al., Nature 391, 51 (1998).

[34] S. Perlmutter et al., Astrophys. J. 517 565, (1999).

[35] R.G. Vishwakarma, Mon. Notice Roy. Astron. Soc. 345, 545 (2003).

[36] M.S. Berman, Nuovo Cim. B 74, 182 (1983).

[37] M.S. Berman and F.M. Gomide, Gen. Relativ. Grav. 20, 191 (1988).

[38] V.B. Johri and K. Desikan, Prammana J. Phys. 42, 473 (1994).

[39] S.D. Maharaj and R. Naidoo, Astrophys. Space Sci. 208, 261 (1993).

[40] G.P. Singh and K. Desikan, Pramana J. Phys., 49, 205 (1997).

[41] F. Rahaman, N. Begum, G. Bag and B.C. Bhui, Astrophys. Space Sci. 299, 211 (2004). 
[42] C.P. Singh and S. Kumar, Int. J. Mod. Phys. D 15, 419 (2006).

[43] C.P. Singh and S. Kumar, Pramana J. Phys.68, 707 (2007).

[44] C.P. Singh and S. Kumar, Astrophys. Space Sci.310, 31 (2007).

[45] S. Kumar and C.P. Singh, Astrophys.Space Sci. 312, 57 (2007).

[46] S. Kumar and C.P. Singh, Int. J. Mod. Phys. A 23, 813 (2008).

[47] ф. Grф n, American J. Phys. 54, 46 (1986).

[48] B. Schwarzschild, Physics Today 40, 17 (1987).
[49] C. Eckart, Phys. Rev. 58, 919 (1940).

[50] R. Maartens, arXiv:astro-ph/9609119

[51] S.W. Hawking and G.R.R. Ellis, The Large Scale Structure of Space Time, Cambridge University Press, Cambridge P88 (1973).

[52] S. Hawking and R. Penrose, Proc. Roy. Soc. 314A, 529 (1970). 